\title{
BMJ Open Economics of abortion: a scoping review protocol
}

\author{
Ernestina Coast, ${ }^{\oplus 1}$ Samantha R Lattof, ${ }^{\oplus 2}$ Yana van der Meulen Rodgers, ${ }^{3}$ \\ Brittany Moore ${ }^{4}$
}

To cite: Coast E, Lattof SR, van der Meulen Rodgers Y, et al. Economics of abortion: a scoping review protocol. BMJ Open

2019;9:e029939. doi:10.1136/ bmjopen-2019-029939

- Prepublication history and additional material for this paper are available online. To view these files, please visit the journal online (http://dx.doi. org/10.1136bmjopen-2019029939).

Received 19 February 2019 Revised 14 June 2019 Accepted 12 July 2019
Check for updates

(C) Author(s) (or their employer(s)) 2019. Re-use permitted under CC BY-NC. No commercial re-use. See rights and permissions. Published by BMJ.

${ }^{1}$ International Development, London School of Economics, London, UK

${ }^{2}$ International Development, London School of Economics and Political Science, London, UK

${ }^{3}$ Women's and Gender Studies, Rutgers The State University of New Jersey, New Brunswick, New Jersey, USA

${ }^{4}$ Scientific and Technical Divison, IPAS, Chapel Hill, North Carolina, USA

Correspondence to Professor Ernestina Coast; e.coas@@Ise.ac.uk

\begin{abstract}
Introduction Abortion is a common feature of people's reproductive lives. However, the economic implications of abortion and policies affecting abortion provision are poorly understood. This scoping review aims to systematically review social science literature for studies that have investigated the impact of abortion care (ie, un/safe abortion, post-abortion care) or abortion policies on economic outcomes at the micro-levels (ie, abortion seekers and their households), meso-levels (ie, communities and health systems) and macro-levels (ie, societies and nation states). Informed by the Preferred Reporting Items for Systematic Reviews and MetaAnalyses (PRISMA) reporting guideline for protocols, this protocol details the scoping review's methodological and analytical approaches.
\end{abstract}

Methods and analysis This scoping review will utilise the PRISMA extension for Scoping Reviews (PRISMA-ScR) tool. Studies reporting on qualitative and/or quantitative data from any world region will be considered. For inclusion, studies must examine one of the following economic outcomes at the micro-levels, meso-levels and/or macrolevels: costs, benefits, impacts and/or value of abortion care or abortion policies. Searches will be conducted in eight electronic databases. We will conduct the searches and application of inclusion/exclusion criteria according to the PRISMA-ScR flow approach. No assessments of items' quality will be made, as the purpose of this scoping review is to synthesise and describe the coverage of the evidence. After extracting all data, we will inductively develop an economic framework around the economics of abortion. The analysis will synthesise the evidence base and identify knowledge gaps on the costs and benefits of abortion to stakeholders at various levels.

Ethics and dissemination Formal ethical approval is not required, as primary data will not be collected in this study. The findings of this study will be disseminated through peer-reviewed publications, conference presentations, and condensed summaries for key stakeholders and partners in the field.

\section{INTRODUCTION}

Abortion is a common feature of people's reproductive lives. An estimated 56 million induced abortions occur annually, ${ }^{1}$ of which $54.9 \%$ (range $49.9 \%-59.4 \%, 90 \% \mathrm{CI}$ ) are unsafe. $^{2}$ Abortion care is a landscape in flux, ${ }^{3}$ with rapid increases in access to and use of pharmaceuticals to induce abortion ${ }^{4}$ and
Strengths and limitations of this study

- This scoping review protocol is the first to focus on the economic costs, benefits and impacts of abortion on individuals, households, communities, health systems and societies.

- We use the Preferred Reporting Items for Systematic Reviews and Meta-Analyses extension for Scoping Reviews tool, the most current guidance on conducting scoping reviews, in order to ensure a systematic approach to searching, screening and reporting.

- This study will search journals from interdisciplinary fields in order to maximise heterogeneity in the results.

- This scoping review may miss studies published outside of journals (eg, book chapters, in-service reports and other grey literature).

- The protocol includes a comprehensive data extraction template that addresses the multiple channels through which abortion can entail economic costs, impacts and benefits.

shifting national and international laws, policies, treaties, protocols and funding provision. ${ }^{56}$ However, the economic implications of abortion-and policies affecting abortion provision ${ }^{7}$-are poorly understood. Relatively little evidence is of use to policymakers and influencers. ${ }^{8}$

A socio-ecological framework identifies three levels of factors-micro (abortion seekers and households), meso (communities and health systems) and macro (societies and nation states) - that help to understand the factors influencing access to abortion services. ${ }^{9}$ These three levels can also be used to consider the consequences of abortion care and abortion policies. There is increased recognition of the scale and consequences of unsafe abortion, including the costs for both women and health systems, in a range of legal settings. ${ }^{10}$ At the macro level, the total cost of post-abortion care to public health systems in many countries is likely to be substantial. Vlassoff $e$ al estimate that US $\$ 171$ million is spent annually to treat abortion complications in Africa. ${ }^{11}$ In Zambia, post-abortion care 
following an unsafe abortion can cost the health system 2.5 times more than safe abortion care. ${ }^{12}$

At the micro-level, inequalities in accessing modern contraception and abortion care have been identified in many settings and are associated with individual characteristics including, but not limited to, economic circumstances. For example, in the USA, low-income women experience more financial and structural barriers to obtaining an abortion than higher-income women. ${ }^{13}$ In Mozambique, women obtaining illegal abortions were more likely to have less education, earn less income, and live in shantytowns compared with women obtaining legal, safe abortions with high hospital fees. ${ }^{14}$ Also related to income constraints, the inability of women living in poverty to afford oral contraception is associated with repeat abortions in France. ${ }^{15}$ The implication is that inequality in using contraceptive methods acts as a determinant affecting abortion behaviour in addition to (or even instead of) inequality in accessing abortion care.

A review of 28 studies on post-abortion care costs in Africa concluded that studies that addressed indirect costs (eg, loss of productivity) were 'conspicuous by their absence. (Woog, p58) ${ }^{8}$ Most research focuses on out-ofpocket expenses that women incur for abortion complications. A Nigerian study of the direct costs for women treated for complications of unsafe abortion estimated that nearly three quarters of costs were shouldered by the woman and/or her household. ${ }^{16}$ A study from Burkina Faso found that the cost of induced abortion was considerably higher than spontaneous abortion, ${ }^{17}$ and this study did not account for any costs incurred by women prior to hospitalisation.

Most studies do not consider the wider economic impact of abortion care seeking, such as opportunity costs (eg, foregone work or education), and few studies include costs incurred throughout the care-seeking process beyond what is paid in hospital. Studies of two Asian countries considered women's loss of time and income. ${ }^{18} 19$ Both found substantial losses for the women and their households. Sundaram et al's study of the costs of abortion care seeking in Uganda calculated the impact of associated expenses on the productivity of women and other family members, as well as households' economic responses to unsafe abortion (eg, sales of assets).$^{20}$ They found that three quarters of women suffered loss of productivity, and over a third experienced deterioration in their economic circumstances following unsafe abortion.

\section{Rationale}

We know relatively little globally about the individual-level economic burden of seeking and procuring abortion. Costs for individuals and their households do not start at point of treatment; rather, costs are incurred directly and indirectly throughout the treatment pathway (eg, transport, food, accommodation, loss of income).$^{21} 22$ Further, costs borne by the poorest women with the least access to contraception and fewest resources are especially high, though infrequently considered. Given these constraints, women's ability to access resources to procure an abortion is important in every setting. Social and emotional support for or against abortion care is linked to whether, and to whom, the pregnancy is disclosed. A friend or partner providing support may influence the location and type of abortion. ${ }^{23}$

Access to financial resources, frequently linked to social support, may be critical to a woman's ability to obtain abortion information and services. In Latin American countries where abortion is illegal, access to financial resources and emotional support were critical for accessing a medically supervised medical abortion in a clandestine clinic. ${ }^{24}$ One quarter of urban Mozambican women who sought a first trimester termination at a public hospital delayed care in order to have sufficient funds to pay user fees. ${ }^{25}$ A pregnancy has short-term and long-term direct and indirect costs for women; these may be exacerbated when the pregnancy is unintended. ${ }^{26}$ Individual circumstances influence whether abortion provides a better outcome for a woman than bearing a child at that time, and women give many reasons for having an abortion. For example, in Bangladesh, women and their husbands described challenging life circumstances (poor health, poverty) that influenced their decisions to terminate. ${ }^{27}$ These examples from Latin America, Africa and Asia bolster the case for making a concerted effort to document the costs to women, households, and societies of seeking and obtaining abortions.

Economists have paid some attention to the relationship between abortion and various economic outcomes at the meso-levels and macro-levels. Much of this literature focuses on the economic impacts of abortion legalisation rather than the pecuniary costs of abortion. Several studies have linked the legalisation of abortion to increases in women's labour supply. For example, Kalist found that by reducing unwanted births, legalisation of abortion in the USA led to increased labour force participation rates for women, especially for single black women. ${ }^{28}$ Bloom et al took this point one step further and found that lower fertility (instrumented by the legalisation of abortion) increases women's labour supply and contributes positively and significantly to gross domestic product (GDP) growth. ${ }^{29}$ Not only do abortion regulations impact women's labour supply, but they also affect occupational mobility. In particular, Targeted Restrictions on Abortion Providers (TRAP) laws in the USA make it more difficult for women to seek an abortion and are linked to increased 'job lock'; consequently, women living in states with TRAP laws are less likely to move between occupations and into higher-paying occupations. ${ }^{30}$ The authors also find that public funding for medically necessary abortions is associated with full-time occupational mobility for women.

The legalisation of abortion is also linked to various measures of children's human capital. Several statistical studies have found positive outcomes for children born after the legalisation of abortion. In a widely cited and somewhat controversial study for the USA, Donohue 
and Levitt found that crime rates across states appear to have dropped as a result of Roe v. Wade. ${ }^{31}$ Children who were born unwanted before the legalisation of abortion grew up in more disadvantaged households and they also grew up to be more disadvantaged as adults. With similar reasoning, Ananat et al found that US children born after the Supreme Court's 1973 Roe v Wade ruling were more likely to graduate from college and less likely to be welfare recipients or single parents. ${ }^{32}$ Children's outcomes may have improved on average because they were more likely to be born into a household in which they were wanted. Romania's abortion ban is associated with worse educational outcomes and labour market achievements of children born after the ban. ${ }^{33}$ And in sub-Saharan Africa, abortion law liberalisation is linked to greater parental investment in girls' schooling, with the rationale that access to abortion lowers the likelihood of a girl child dropping out of school in the event of an unplanned pregnancy. ${ }^{34}$

\section{Scoping review objectives}

We lack synthesis of the known economic consequencesat a variety of scales-of abortion care and abortion policies. We aim to systematically review social science literature for studies that have investigated the impact of abortion care (ie, un/safe abortion, post-abortion care) and abortion policies on economic outcomes at the micro-levels, meso-levels and macro-levels. To achieve this objective, the scoping review will answer the following question:

What are the economic costs, benefits and consequences of abortion care and policies at the micro-levels, meso-levels and macro-levels?

Informed by the Preferred Reporting Items for Systematic Reviews and Meta-Analyses (PRISMA) reporting guideline for protocols, ${ }^{35}$ this protocol details our preplanned methodological and analytical approaches.

\section{METHODS AND ANALYSIS}

Since we are interested in examining what is known about the economic consequences of abortion care and abortion policies, and we expect to uncover varied evidence on this topic, we will conduct a scoping review. Like systematic reviews, scoping reviews use a systematic approach to searching, screening, and reporting. ${ }^{36}$ Our scoping review will utilise the PRISMA extension for Scoping Reviews (PRISMA-ScR) tool. Published in October 2018, this innovative checklist is the most up-to-date guidance on conducting scoping reviews. ${ }^{36}$

\section{Inclusion/exclusion criteria}

Studies published in peer-reviewed journals on induced abortion and/or post-abortion care in any world region will be considered, provided that they report on qualitative and/or quantitative data. (These data may include policy and legal documents.) More specifically, these data must examine one of the following economic (Our approach includes economic outcomes related to human capital and women's reproductive labour that are not directly quantified in monetary terms. Such outcomes could include education, mothering and care work.) outcomes at the micro-levels, meso-levels and/or macro-levels:

- Economic costs ('Economic costs' refer to the amount paid to obtain abortion care or adverse financial outcomes resulting from the implementation of abortion policies.) of abortion care or abortion policies.

- Economic benefits ('Economic benefits' refer to the advantages or profits gained from receiving abortion care or from the implementation of abortion policies.) of abortion care or abortion policies.

- Economic impacts ('Economic impacts' refer to the economic effect or influence of abortion care or abortion policies.) of abortion care or abortion policies.

- Economic value ('Economic value' refers to the importance, worth, welfare gains, or utility from receiving abortion care or of the implementation of abortion policies.) of abortion care or abortion policies.

As indicated in the Populations, Interventions, Control, Outcomes, Timeframe, Setting (PICOTS) criteria in table 1, the screening criteria differ depending on the level (micro, meso, macro) at which the study occurred. Specifically, the population changes to account for the fact that we are examining evidence from the individual level to the national level.

Items must be published in peer-reviewed journals or in the National Bureau of Economic Research's (NBER) peer-reviewed working paper series, which is considered to be a gold standard in the field of economics. Any NBER working paper that is subsequently published in a peer-reviewed journal will only be considered in its final published version.

Items must be published in English, French, Spanish, Dutch, or German. This review includes studies published from 1 September 1994 to 15 January 2019.

Items will be excluded if they focus on missed abortion, threatened abortion, or miscarriage. In addition, we will exclude policy briefs, books, book chapters, editorials, commentaries and published or unpublished reports from governments and other agencies. By limiting included items to peer-reviewed journal articles or NBER working papers that have been subjected to the scrutiny of other experts in the field, we increase the possibility that our scoping review will include items with lower likelihood of the inclusion of errors. ${ }^{37}$

\section{Search strategy and terms}

After first assessing electronic databases for their relevance and coverage of the literature, we selected eight electronic databases for searching:

- Cumulative Index to Nursing and Allied Health.

- EconLit.

- Excerpta Medica Database (EMBASE).

- International Bibliography of the Social Sciences.

- JSTOR.

- PubMed. 


\begin{tabular}{|c|c|c|c|}
\hline PICOTS & Micro-level & Meso-level & Macro-level \\
\hline Populations & $\begin{array}{l}\text { Girls and women who obtained } \\
\text { abortions or post-abortion } \\
\text { care and members of their } \\
\text { households }\end{array}$ & $\begin{array}{l}\text { Communities and health } \\
\text { systems in which girls and } \\
\text { women obtain abortions or } \\
\text { post-abortion care }\end{array}$ & $\begin{array}{l}\text { Societies and nation states in } \\
\text { which girls and women obtain } \\
\text { abortions or post-abortion care }\end{array}$ \\
\hline Interventions & \multicolumn{3}{|c|}{ Induced abortion (safe/unsafe), post-abortion care and/or abortion policies } \\
\hline Outcomes & \multicolumn{3}{|c|}{$\begin{array}{l}\text { Quantitative or qualitative data on: } \\
\text { - Economic costs of abortion care or abortion policies. } \\
\text { Economic impacts of abortion care or abortion policies. } \\
\text { Economic benefits of abortion care or abortion policies. } \\
\text { - Economic value of abortion care or abortion policies. }\end{array}$} \\
\hline
\end{tabular}

- ScienceDirect.

- Web of Science.

These sources will be searched using combinations of relevant search terms that we developed and tested for sensitivity in advance of the scoping review. The terms, detailed in table 2, will be adapted to the basic search particulars (eg, wildcards $(*)$ and truncations, capacity for complex searches) of each electronic database. We will supplement these searches with expert-recommended articles. To obtain these articles, we will develop a standardised email asking for suggested articles that we will send to a list of abortion researchers. Any suggested articles will be incorporated into our PRISMA flowchart.

The impact terms are broad enough to capture numerous terms that are directly related to economic outcomes, such as health, education and income. These outcomes capture the mechanisms through which

\section{Table 2 Search terms and their combinations}

\begin{tabular}{|c|c|c|}
\hline Abortion terms & Economic terms & $\begin{array}{l}\text { Impact } \\
\text { terms }\end{array}$ \\
\hline abort $^{*}$ & cost $^{\star}$ & cost $^{*}$ \\
\hline $\begin{array}{l}\text { termination of } \\
\text { pregnancy }\end{array}$ & econom* & benefit* $^{\star}$ \\
\hline $\begin{array}{l}\text { terminate } \\
\text { pregnancy }\end{array}$ & price* $^{*}$ & value $^{*}$ \\
\hline $\begin{array}{l}\text { pregnancy } \\
\text { termination }\end{array}$ & financ* $^{*}$ & impact $^{\star}$ \\
\hline $\begin{array}{l}\text { pregnancy } \\
\text { terminations }\end{array}$ & resource $^{\star}$ & \\
\hline postabortion & $\mathrm{fee}^{*}$ & \\
\hline \multirow[t]{6}{*}{ post-abortion } & $\operatorname{tax}^{*}$ & \\
\hline & expenditure* & \\
\hline & GDP & \\
\hline & gross domestic product & \\
\hline & pay* & \\
\hline & expens* & \\
\hline
\end{tabular}

abortions may have economic consequences for individuals, households, and even the macroeconomy. The impact terms 'value' and 'benefit' are not meant to be mutually exclusive; they are common terms in studies on the economics of abortion. By including them both in the screening process, we are less likely to miss a relevant study.

\section{Screening process}

To ensure compatibility with the standards expected of a scoping review for peer-reviewed publication, we will conduct the searches and application of inclusion/ exclusion criteria according to the PRISMA-ScR flow approach. ${ }^{36}$ No assessments of items' quality will be made, as the purpose of this scoping review is to synthesise and describe the coverage of the evidence.

Once the searches are conducted, citation abstracts for all items will be exported into EndNote for screening. After removing duplicates, the remaining items will be screened for inclusion, initially on the basis of title and abstract (TIAB). When inclusion or exclusion cannot be determined on the basis of TIAB, the person screening the item will move the item forward for full-text screening. To assure quality in TIAB screening, EC, EZ, SL and YR will simultaneously screen 100 randomly selected items for inclusion. Based on our results, we will adjust the inclusion/exclusion criteria as necessary. If the results of our individual screenings differ, we will screen an additional 100 randomly selected items for inclusion on TIAB based on the refined inclusion/exclusion criteria. The process will be repeated until we reach agreement. The remaining items will then be divided among the authors, CP, and EZ for full-text screening. Following the full-text screening, studies recommended for exclusion will be reviewed by a second researcher to ensure consistency in the application of exclusion criteria.

\section{Data extraction}

BM, CP, EC, EZ, SL and YR will simultaneously extract data into Excel for five randomly selected studies in order 
to assure quality in data extraction. Following this check for quality assurance, which will be repeated until agreement is reached, we will divide the remaining included studies for data extraction. Data will be extracted on the following categories:

- Background information (eg, author, date, setting, study objective).

- Population.

- Details of relevant outcomes (both quantitative and qualitative) at the micro-levels, meso-levels and macro-levels.

- Financial cost (the amount paid to obtain abortion care, including transportation costs and opportunity costs when relevant) or adverse financial outcomes from abortion policies.

- Impact (the effect or influence of abortion care or abortion policies).

- Benefit (advantages or profits gained from receiving abortion care or implementing abortion policies).

- Value (the importance, worth, welfare gains, or utility of receiving abortion care or implementing abortion policies).

- Secondary outcome data on abortion-related stigma, discrimination and exclusion.

- Context in which the study was conducted (eg, legal status of abortion, culture, gender norms).

Since this scoping review intends to synthesise and describe the coverage of the evidence, we will not assess the risk of bias of individual studies. A detailed data extraction template is available in online supplementary appendix A, and the accompanying codebook is available in online supplementary appendix B.

\section{Data synthesis}

After extracting all data, we will inductively develop an economic framework around the economics of abortion. The analysis will synthesise the evidence base and identify knowledge gaps on the costs, impacts and benefits of abortion to stakeholders at the micro-levels, meso-levels and macro-levels. At the micro-level, we will provide a comprehensive examination of women's decision-making around contraceptive use, fertility and abortion. The framework is based on a set of economics tools related to marginal cost and risk avoidance, and it generates a number of scenarios showing how changes in the cost and availability of modern contraceptive methods and access to safe abortion can impact the health, well-being, and economic status of women and their households.

At the meso-level, we will consider the economic costs and impacts of abortion care to communities and health systems and also how health systems bear the costs of abortion restrictions that may increase the number of women seeking post-abortion care.

Finally, at the macro-level, we will explore how liberalising or restricting abortion legislation impacts broad aggregates such as women's labour force participation, women's education, investment in children's human capital, and economic growth. Women's ability to control the timing and number of births through access to modern contraception is linked to higher maternal age at first birth, fewer children and longer birth intervals. These factors are all linked to improved maternal health, which not only helps women but also has repercussions for healthcare costs and the overall macroeconomy through investment in women's human capital and that of their children. Furthermore, the relationship between women's socioeconomic status and the likelihood of using abortion enables us to infer the types of labour market opportunities for women who had abortions relative to those who did not.

We will report the data using a systematic narrative synthesis in which the results are presented narratively and organised thematically, supplemented with tables of descriptive statistics on included studies and their outcomes.

\section{Patient and public involvement}

The design of this scoping review protocol did not involve patients. However, patients' experiences are central to the research question and outcome measures. At the micro-level, our analyses will synthesise the evidence base and identify evidence gaps on the costs and benefits of abortion to girls and women seeking abortions and their households.

\section{FINAL SEARCH STRATEGY BY DATABASE}

The full electronic search strategies for all databases, including limits used, appear below.

\section{Cumulative Index to Nursing and Allied Health Search strategy \\ We will search all sets of search terms (table 2). \\ Search options \\ - Search mode: Boolean/phrase. \\ - Limit results: - Published date: September 1994 to January 2019.}

\section{Search terms}

(abort* OR 'termination of pregnancy' OR 'terminate pregnancy' OR 'pregnancy termination' OR 'pregnancy terminations' OR postabortion OR post-abortion) AND (cost* OR econom* OR price* OR financ* OR fee* OR tax* OR expenditure* OR GDP OR 'gross domestic product' OR pay* OR expens*) AND (cost* OR benefit* OR value* OR impact*).

\section{EconLit}

\section{Search strategy}

We will search all sets of search terms (table 2).

\section{Search options}

- Search mode: Boolean/phrase.

- Limit results:

- Published date: September 1994 to January 2019. 


\section{Search terms}

(abort* OR 'termination of pregnancy' OR 'terminate pregnancy' OR ‘pregnancy termination' OR ‘pregnancy terminations' OR postabortion OR post-abortion) AND (cost* OR econom* OR price* OR financ* OR fee* OR tax* OR expenditure* OR GDP OR 'gross domestic product' OR pay* OR expens*) AND (cost* OR benefit* OR value* OR impact*).

\section{Excerpta Medica Database}

Search strategy

We will search modified sets of search terms (table 2) using the multifield search. Since EMBASE does not recognise the use of quotation marks for multi-word phrases, searches would include results with the word 'of' (from 'termination of pregnancy'). To exclude the 'of' from searches, we will modify the abortion-related search terms, as detailed below. Results will be aggregated with duplicates removed before they are added to EndNote.

\section{Search options}

- Limit results:

- Publication year: 1994-15 January 2019.

\section{Search terms}

(abort* OR postabortion OR post-abortion OR (terminat* AND pregnancy)) AND (cost* OR econom* OR price* OR financ* OR fee* OR tax* OR expenditure* OR GDP OR 'gross domestic product' OR pay* OR expens*) AND (cost* OR benefit* OR value* OR impact*).

\section{International Bibliography of the Social Sciences \\ Search strategy}

We will search all sets of search terms (table 2) using the advanced search feature. Test searches returned numerous extraneous results; searches will be limited to abstract and title, since all results will be screened against TIAB.

\section{Search options}

- Limit results:

- Publication date: 1 September 1994-15 January 2019.

- Language:

- English

- French.

- Spanish.

- Dutch.

- German.

- Source type: Scholarly journals.

- Peer-reviewed.

- Document type: Article (including original research articles), case report, case study, clinical trial, comparative study, correction/retraction, essay, evaluation studies, literature review, report, review, technical report.

- Exclude duplicate items.
Search terms

(ti(abort* OR 'termination of pregnancy' OR 'terminate pregnancy' OR 'pregnancy termination' OR 'pregnancy terminations' OR postabortion OR post-abortion) AND ti((cost* OR econom* OR price* OR financ* OR fee* OR tax* OR expenditure* OR GDP OR 'gross domestic product' OR pay* OR expens*)) AND ti((cost* OR benefit* OR value* OR impact*)) AND la.exact('German' OR 'Spanish' OR 'English' OR 'French' OR 'Dutch') AND (rtype. exact('Journal Article' OR 'Article' OR 'Review' OR 'Comparative Study' OR 'Case Study' OR 'Literature Review' OR 'Case_Study' OR 'Evaluation Studies' OR 'Research Article' OR 'Case Reports' OR 'article' OR 'JOURNAL ARTICLE' OR 'Original Research Articles' OR 'review' OR 'Review article' OR 'Clinical Trial' OR 'Research article' OR 'CLINICAL TRIAL' OR 'Clinical Trial, Phase I' OR 'Literature_Review' OR 'Case Report') AND stype.exact('Scholarly Journals') AND PEER(yes))) OR (ab(abort* OR 'termination of pregnancy' OR ‘terminate pregnancy' OR 'pregnancy termination' OR ‘pregnancy terminations' OR postabortion OR post-abortion) AND ab((cost* OR econom* OR price* OR financ* OR fee* OR tax* OR expenditure* OR GDP OR 'gross domestic product' OR pay* OR expens*)) AND ab((cost* OR benefit* OR value* OR impact*)) AND la.exact('German' OR 'Spanish' OR 'English' OR 'French' OR 'Dutch' OR 'English' OR 'Spanish' OR 'French' OR 'German' OR 'Dutch') AND (rtype.exact('Journal Article' OR 'Article' OR 'Review' OR 'Comparative Study' OR 'Case Study' OR 'Literature Review' OR 'Case_Study' OR 'Evaluation Studies' OR 'Research Article' OR 'Case Reports' OR 'article' OR 'JOURNAL ARTICLE' OR 'Original Research Articles' OR 'review' OR 'Review article' OR 'Clinical Trial' OR 'Research article' OR 'CLINICAL TRIAL' OR 'Clinical Trial, Phase I' OR 'Literature_Review' OR 'Case Report') AND stype.exact('Scholarly Journals') AND la. exact( 'ENG' OR 'SPA' OR 'FRE' OR 'GER' OR 'DUT') AND PEER(yes))).

\section{JSTOR}

Search strategy

We will search all search terms (table 2). Since JSTOR does not permit searches of the length necessary to capture all three sets of search terms in one search, we will conduct three separate searches, as detailed below. Searches will be conducted using the advanced search feature and 'all content' access type. Results will be aggregated with duplicates removed before they are added to EndNote.

\section{Search options}

Limit results:

- Content type: Articles.

- Publication date: From September 1994 to 15 January 2019.

- Narrowed by discipline: 
- Economics.

- Feminist and women's studies.

- Health policy.

- Health sciences.

- Population studies.

- Public health.

Search terms for Search \#1

(abort* OR 'termination of pregnancy' OR 'terminate pregnancy' OR 'pregnancy termination' OR 'pregnancy terminations' OR postabortion OR post-abortion) AND (cost* OR econom* OR price*) AND (cost* OR benefit* OR value* OR impact*).

\section{Search terms for Search \#2}

(abort* OR 'termination of pregnancy' OR 'terminate pregnancy' OR 'pregnancy termination' OR 'pregnancy terminations' OR postabortion OR post-abortion) AND (financ* OR fee* OR tax* OR expenditure*) AND (cost* OR benefit* OR value* OR impact*).

\section{Search terms for Search \#3}

(abort* OR 'termination of pregnancy' OR 'terminate pregnancy' OR 'pregnancy termination' OR 'pregnancy terminations' OR postabortion OR post-abortion) AND (GDP OR 'gross domestic product') AND (cost* OR benefit* OR value* OR impact*).

\section{Pubmed}

\section{Search strategy}

We will search all sets of search terms (table 2) using the advanced search builder. Test searches returned numerous extraneous results; we will limit searches to TIAB, since these results will be screened against TIAB.

\section{Search options}

Limit results:

- Publication dates: From 1 September 1994 to 15 January 2019.

- Language:

- German.

- Dutch.

- Spanish.

- French.

- English.

\section{Search terms}

( ( (abort*[Title/Abstract] OR 'termination of pregnancy'[Title/Abstract] OR 'terminate pregnancy'[Title/Abstract] OR 'pregnancy termination'[Title/ Abstract] OR 'pregnancy terminations' [Title/Abstract] OR postabortion[Title/Abstract] OR post-abortion[Title/Abstract])) AND (cost*[Title/Abstract] OR econom*[Title/Abstract] OR price*[Title/Abstract] OR financ*[Title/Abstract] OR fee*[Title/Abstract] OR tax*[Title/Abstract] OR expenditure*[Title/ Abstract] OR GDP[Title/Abstract] OR 'gross domestic product'[Title/Abstract] OR pay*[Title/Abstract] OR expens*[Title/Abstract])) AND (cost*[Title/Abstract]
OR benefit*[Title/Abstract] OR value*[Title/Abstract] OR impact*[Title/Abstract])).

\section{ScienceDirect}

Search strategy

Since this database does not support wildcards $(*)$ or more than eight Boolean connectors per field, we will search a modified set of abortion-related and economic-related search terms (table 2) using the advanced search feature. We will conduct three searches, as detailed below. Results will be aggregated with duplicates removed before they are added to EndNote.

\section{Search options}

- Limit results:

- Article types: Review articles, research articles, case reports, data articles.

- Year(s): 1994-2019.

\section{Search terms for Search \#1}

- Find articles with these terms: cost OR costs OR economic OR economics OR prices OR price OR finance OR fees OR fee Title, abstract or keywords: abortion OR 'termination of pregnancy' OR 'terminate pregnancy' OR 'pregnancy termination' OR 'pregnancy terminations' OR postabortion OR post-abortion.

\section{Search terms for Search \#2}

- Find articles with these terms: GDP OR 'gross domestic product' OR pay OR payment OR payments OR expenses OR expense OR expensive OR tax.

- Title, abstract or keywords: abortion OR 'termination of pregnancy' OR 'terminate pregnancy' OR 'pregnancy termination' OR 'pregnancy terminations' OR postabortion OR post-abortion.

\section{Search terms for Search \#3}

- Find articles with these terms: taxes OR expenditure OR expenditures.

- Title, abstract or keywords: abortion OR 'termination of pregnancy' OR 'terminate pregnancy' OR 'pregnancy termination' OR 'pregnancy terminations' OR postabortion OR post-abortion.

\section{Web of science}

\section{Search strategy}

We will search all sets of search terms (table 2) using the advanced search feature and topic (TS) field tag.

\section{Search options}

- Limit results:

- Article types: Article, abstract of published item, early access.

- Year(s): 1994-2019.

- Language:

- English.

- French.

- Spanish. 
- Dutch.

- German.

\section{Search terms}

(abort* OR 'termination of pregnancy' OR 'terminate pregnancy' OR 'pregnancy termination' OR 'pregnancy terminations' OR postabortion OR post-abortion) AND (cost* OR econom* OR price* OR financ* OR fee* OR tax* OR expenditure* OR GDP OR 'gross domestic product' OR pay* OR expens*) AND (cost* OR benefit* OR value* OR impact*).

\section{ETHICS AND DISSEMINATION}

Formal ethical approval is not required, as primary data will not be collected in this study.

The findings of this scoping review will be used to create a framework to articulate the economic value, impact and costs of abortion. This framework can be used for advocacy efforts in the field to increase access to cost-effective health services. This framework can also be used to inform future research efforts to address current evidence gaps in the field. The findings of this scoping review will be published in a peer-reviewed journal and circulated through relevant mailing lists and social media platforms. The findings will also be disseminated through conference presentations and as condensed summaries for key stakeholders and partners.

If we need to amend this protocol following its publication, we will provide the date of each amendment, describe the change(s), and report the rationale for the change(s) in future publications arising from this protocol.

Acknowledgements We wish to thank Elaine Zundl (EZ) for her input on the inclusion/exclusion criteria; Cheri Poss (CP) and Alli Buehler for reviewing an earlier draft of this protocol; and Cheri Poss for her contributions to the wider discussions around this research.

Contributors BM conceived the idea for the review, with inputs from EC and YMR. EC, SRL and YMR designed and drafted the scoping protocol. EC and YMR drafted the introduction section, SRL drafted the methods section, and BM drafted the ethics and dissemination section. All authors contributed to subsequent revisions and approved the protocol prior to its submission. EC is the guarantor.

Funding This work was supported by the Netherlands Ministry of Foreign Affairs, activity number 28438. This funder had no role in the design and development of the study protocol or the decision to publish.

Competing interests None declared.

Patient consent for publication Not required.

Provenance and peer review Not commissioned; externally peer reviewed.

Open access This is an open access article distributed in accordance with the Creative Commons Attribution Non Commercial (CC BY-NC 4.0) license, which permits others to distribute, remix, adapt, build upon this work non-commercially, and license their derivative works on different terms, provided the original work is properly cited, appropriate credit is given, any changes made indicated, and the use is non-commercial. See: http://creativecommons.org/licenses/by-nc/4.0/.

\section{REFERENCES}

1. Sedgh G, Bearak J, Singh S, et al. Abortion incidence between 1990 and 2014: global, regional, and subregional levels and trends. Lancet 2016;388:258-67.
2. Ganatra B, Gerdts C, Rossier C, et al. Global, regional, and subregional classification of abortions by safety, 2010-14: estimates from a Bayesian hierarchical model. Lancet 2017;390:2372-81.

3. Coast E, Murray SF. "These things are dangerous": Understanding induced abortion trajectories in urban Zambia. Soc Sci Med 2016;153:201-9.

4. Kapp N, Blanchard K, Coast E, et al. Developing a forward-looking agenda and methodologies for research of self-use of medical abortion. Contraception 2018;97:184-8.

5. Barot S. When antiabortion ideology turns into foreign policy: how the global GAGE rule erodes health, ethics and democracy. Guttmacher Policy Review 2017;20:73-7.

6. Barot $\mathrm{S}$. The benefits of investing in international family planning and the price of slashing funding. Guttmacher Policy Review 2017;20:1-4

7. YvdM R. The global gag rule and women's reproductive health: rhetoric versus reality. Oxford: Oxford University Press, 2019.

8. Woog V, Singh S, Bankole A. A review of the evidence on the cost of post-abortion care in Africa. In: Lule E, Singh S, Chowdhury SA, eds. Fertility regulation behaviors and their costs: contraception and unintended pregnancies in Africa and eastern Europe \& central Asia. Washington: World Bank, 2007: 40-92.

9. Coast E, Norris AH, Moore AM, et al. Trajectories of women's abortion-related care: a conceptual framework. Soc Sci Med 2018;200:199-210.

10. Singh S, Maddow-Zimet I. Facility-based treatment for medical complications resulting from unsafe pregnancy termination in the developing world, 2012: a review of evidence from 26 countries. BJOG: Int J Obstet Gy 2016;123:1489-98.

11. Vlassoff M, Walker D, Shearer J, et al. Estimates of health care system costs of unsafe abortion in Africa and Latin America. Int Perspect Sex Reprod Health 2009;35:114-21.

12. Parmar D, Leone T, Coast E, et al. Cost of abortions in Zambia: a comparison of safe abortion and post abortion care. Glob Public Health 2017;12:236-49.

13. Ostrach B, Cheyney M. Navigating social and institutional obstacles: low-income women seeking abortion. Qualitative health research 2014;240::1006-17. 7.

14. Machungo F, Zanconato G, Bergström S. Socio-Economic background, individual cost and hospital care expenditure in cases of illegal and legal abortion in Maputo. Health Soc Care Community 1997;5:71-6.

15. Alouini S, Uzan M, Méningaud JP, et al. Knowledge about contraception in women undergoing repeat voluntary abortions, and means of prevention. Eur J Obstet Gynecol Reprod Biol 2002;104:43-8.

16. Bankole A, Singh S, Vlassoff M, et al. Estimating the cost of postabortion care in Nigeria: a case study. Fertility Regulation Behaviors and Their Costs: Contraception and Unintended Pregnancies in Africa and Eastern Europe \& Central Asia. Washington, DC: World Bank, 2007: 65-92.

17. Ilboudo PGC, Greco G, Sundby J, et al. Costs and consequences of abortions to women and their households: a cross-sectional study in Ouagadougou, Burkina Faso. Health Policy Plan 2015;30:500-7.

18. Narkavonnakit T, Bennett T. Health consequences of induced abortion in rural northeast Thailand. Stud Fam Plann 1981;12:58-65.

19. Potdar R, Fetters T, Phirun L. Initial loss of productive days and income among women seeking induced abortion in Cambodia. $J$ Midwifery Womens Health 2008;53:123-9.

20. Sundaram A, Vlassoff M, Bankole A, et al. The economic cost of unsafe abortion: a study of post-abortion care patients in Uganda. XXVII IUSSP conference. Republic of Korea: Busan, 2013.

21. Leone T, Coast E, Parmar D, et al. The individual level cost of pregnancy termination in Zambia: a comparison of safe and unsafe abortion. Health Policy Plan 2016;31:825-33. 7.

22. Edejer TT. Making choices in health: WHO guide to cost-effectiveness analysis. World Health Organization, 2003.

23. Conkling K, Karki C, Tuladhar $\mathrm{H}$, et al. A prospective open-label study of home use of mifepristone for medical abortion in Nepal. Int $J$ Gynaecol Obstet 2015;128:220-3.

24. Zamberlin N, Romero M, Ramos S. Latin American women's experiences with medical abortion in settings where abortion is legally restricted. Reprod Health 2012;9:34.

25. Mitchell EMH, Kwizera A, Usta M, et al. Choosing early pregnancy termination methods in urban Mozambique. Soc Sci Med 2010;71:62-70.

26. Gipson JD, Koenig MA, Hindin MJ. The effects of unintended pregnancy on infant, child, and parental health: a review of the literature. Stud Fam Plann 2008;39:18-38. 
27. Gipson JD, Hindin MJ. "Having another child would be a life or death situation for her": understanding pregnancy termination among couples in rural Bangladesh. Am J Public Health 2008;98:1827-32.

28. Kalist DE. Abortion and female labor force participation: evidence prior to roe V. Wade. J Labor Res 2004;25:503-14.

29. Bloom DE, Canning D, Fink G, et al. Fertility, female labor force participation, and the demographic dividend. Journal of Economic Growth 2009;14:79-101.

30. Bahn K, Kugler A, Mahoney M, et al. Linking reproductive health care access to labor market opportunities for women. Washington, DC: Center for American Progress, 2018.

31. Donohue JJ, Levitt SD. The impact of legalized abortion on crime. $Q$ $J$ Econ 2001;116:379-420.

32. Ananat EO, Gruber J, Levine PB, et al. Abortion and selection. Rev Econ Stat 2009;91:124-36.
33. Pop-Eleches $\mathrm{C}$. The impact of an abortion ban on socioeconomic outcomes of children: evidence from Romania. J Polit Econ 2006;114:744-73.

34. Azarnert LV. Abortion and human capital accumulation: a contribution to the understanding of the gender gap in education. Scott $J$ Polit Econ 2009;56:559-79.

35. Moher D, Shamseer L, Clarke M, et al. Preferred reporting items for systematic review and meta-analysis protocols (PRISMA-P) 2015 statement. Syst Rev 2015;4:1.

36. Tricco AC, Lillie E, Zarin W, et al. Prisma extension for scoping reviews (prisma-scr): checklist and explanation. Ann Intern Med 2018;169:467-73.

37. Kelly J, Sadeghieh T, Adeli K. Peer review in scientific publications: benefits, critiques, \& a survival guide. EJIFCC 2014:25:227-43. 